\title{
Significados atribuídos à morte segundo a perspectiva de profissionais de saúde da área de oncologia
}

\author{
Meanings attributed to death from the perspective of oncology healthcare professionals
}

Significados atribuidos a la muerte desde la perspectiva de profesionales de salud del área de oncología

\author{
José Henrique da Silva Cunhal @ ; Lúcia Aparecida Ferreira" @; Heloísa Cristina Figueiredo Frizzo" @ ;

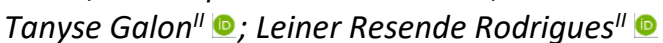 \\ 'Universidade de São Paulo, Ribeirão Preto, SP, Brasil, "Universidade Federal do Triângulo Mineiro, Uberaba, MG, Brasil
}

\begin{abstract}
RESUMO
Objetivo: compreender a construção dos significados da morte pelos profissionais de saúde frente ao cuidado à pessoa com câncer. Método: estudo qualitativo realizado em ambiente online, com a participação de 34 profissionais de saúde. Foram realizadas entrevistas semiestruturadas, interpretadas segundo análise temática. Resultados: duas categorias analíticas emergiram dos dados, indicando que os significados atribuídos à morte pelos participantes foram: como um processo inerente ao ciclo da vida humana, passagem para a outra vida, e fim do sofrimento tanto do paciente quanto dos profissionais de saúde. As dificuldades que apontaram em lidar com a morte se referem à falta de suporte psicológico, escassez de conhecimento sobre morte, cuidados paliativos e comunicação de más notícias. Conclusão: os profissionais construíram diferentes significados à morte de seus pacientes, tais como um processo natural da vida, vontade de um ser superior, passagem para uma nova existência e término do sofrimento dessas pessoas.
\end{abstract}

Descritores: Morte; Neoplasias; Pessoal de Saúde; Atitude Frente a Morte; Prática Profissional.

\begin{abstract}
Objective: to understand the construction of meanings of death by healthcare professionals caring for people with cancer. Method: this qualitative study was carried out in an online environment through semi-structured interviews of 34 health professionals, which were interpreted using thematic analysis. Results: two analytical categories emerged from the data, indicating that the meanings attributed to death by the participants were: a process inherent in the human life cycle, a transition to the next life, and an end to the suffering of both patient and health professionals. The difficulties they pointed to in dealing with death related to lack of psychological support, lack of knowledge about death, palliative care, and communicating bad news. Conclusion: the professionals constructed different meanings to their patients' deaths: a natural process of life, the will of a higher being, a transition to a new existence, and an end to these people's suffering.

Descriptors: Death; Neoplasms; Health Personnel; Attitude to Death; Professional Practice.

\section{RESUMEN}

Objetivo: comprender la construcción de los significados de la muerte desde el punto de vista de los profesionales de la salud ante el cuidado a la persona con cáncer. Metodología: estudio cualitativo realizado en un entorno online, con la participación de 34 profesionales de la salud. Se realizaron entrevistas semiestructuradas, interpretadas según análisis temático. Resultados: de los datos surgieron dos categorías analíticas que indicaron que los significados atribuidos a la muerte por los participantes fueron: como u proceso inherente al ciclo de vida humano, transición a otra vida y fin al sufrimiento tanto del paciente como de los profesionales de la salud. Las dificultades que señalaron para afrontar la muerte se refieren a la falta de apoyo psicológico, escasez de conocimiento sobre la muerte, cuidados paliativos y comunicación de malas noticias. Conclusión: los profesionales han construido diferentes significados para la muerte de sus pacientes: un proceso natural de la vida, la voluntad de un ser superior, un paso a una nueva existencia y el fin del sufrimiento de estas personas.

Descriptores: Muerte; Neoplasias; Personal de Salud; Actitud Frente a la Muerte; Práctica Profesional.
\end{abstract}

\section{INTRODUÇÃO}

O câncer, doença agressiva e complexa, merece particular importância no olhar das políticas e das organizações de saúde em nível global, por ser uma das principais causas de morte por doença em todos os países do mundo ${ }^{1}$.

Em relação à morte, profissionais da área da saúde ainda enfrentam dificuldades para superar uma visão estritamente curativa e reconhecer quando o paciente se encontra fora das possibilidades terapêuticas de cura, com possibilidade de morrer em virtude de sua doença ${ }^{2}$. Em sua formação, eles são preparados para, sobretudo, recuperar a saúde e preservar a vida; e a ocorrência da morte de seus pacientes os faz refletir sobre sua própria finitude e de seus familiares ${ }^{3}$. 
A dificuldade em lidar com a morte pode ocasionar adoecimento nos profissionais de saúde que vivenciam a morte de seus pacientes em sua atividade laboral ${ }^{2,4}$. Tais dificuldades podem influenciar na assistência e nos cuidados prestados às pessoas, especialmente àquelas que se encontram em cuidados paliativos, podendo ocorrer um afastamento e uma relação estritamente profissional com esses indivíduos, como uma forma de proteção, por não conseguirem lidar com essas experiências de perda ${ }^{5,6}$.

O processo de morte do paciente com câncer pode gerar desenvolvimento de luto no profissional de saúde. Dentre as abordagens direcionadas à compreensão do luto, destaca-se neste estudo a perspectiva da teoria da construção do significado ${ }^{7}$. Essa teoria é embasada no construtivismo, no qual a pessoa é vista como uma construtora ativa de significados $^{8}$. Nessa perspectiva, compreende-se que os sistemas humanos, do indivíduo singular às organizações e comunidades, procuram constantemente definir seu lugar no mundo e dar significado às suas experiências. Por conseguinte, é possível desvelar a realidade da morte ou da perda para cada pessoa, em vez de afirmar que a morte tem um significado universal para todos os indivíduos ${ }^{9}$.

A ocorrência da morte para os profissionais de saúde não é um fenômeno vivido de forma natural, mas acompanhado de inúmeras dificuldades, sugerindo que o sofrimento resultante do contato rotineiro com situações de terminalidade quase sempre é velado e silenciado ${ }^{10}$.

Apesar das diferentes categorias profissionais de saúde que cuidam de pessoas com câncer vivenciarem a morte de seus pacientes diariamente, os sentidos e os significados que irão construir e atribuir a esse acontecimento inevitável da vida serão diferentes e únicos. Com o desvelar dessas experiências, será possível sensibilizar a gestão dos serviços de atenção oncológica, e os serviços de saúde de modo geral, a fim de sugerir caminhos para o desenvolvimento de ações que visem acolhê-los e capacitá-los a respeito de assuntos que envolvam a finitude da vida, para que possam lidar com a morte de seus pacientes. Em face do exposto, questionou-se: qual é a construção dos significados da morte para os profissionais de saúde, frente ao cuidado à pessoa com câncer? Este estudo objetivou compreender a construção dos significados da morte para os profissionais de saúde frente ao cuidado à pessoa com câncer.

\section{REFERENCIAL TEÓRICO}

Adotou-se a construção de significados perante o luto, embasada no construtivismo ${ }^{8}$. Essa perspectiva defende o papel ativo das pessoas na produção de suas experiências humanas e na atribuição de significado em face a essas experiências, recorrendo à linguagem para dar significado e expressar dimensões importantes da sua experiência no mundo ${ }^{8}$.

Para Neimeyer ${ }^{11}$, a construção de significados é um processo ativo diante da perda. $O$ autor também ressalta que a narrativa escolhida pelo enlutado para contar a sua história de perda e os significados a ela atribuídos são de vital importância para o desenvolvimento de um luto saudável, pois é através dela que a pessoa se organiza e dá significado ao mundo.

\section{MÉTODO}

Trata-se de um estudo descritivo e exploratório com abordagem qualitativa ${ }^{12}$ que foi realizado em ambiente online, que é um modo de realizar investigações qualitativas no contexto de trabalho de pesquisa na internet que permite ao pesquisador utilizar ferramentas de comunicação (chats ou videoconferências) como recursos para o contato e a interação com os participantes da pesquisa ${ }^{13}$.

Participaram desse estudo 34 profissionais de saúde, atuantes em contexto hospitalar no cuidado à pessoa com câncer. Os critérios de inclusão foram: ser profissional de saúde atuante na assistência a pacientes com câncer, ter vivenciado a morte dessas pessoas e ter aceitado participar da pesquisa a partir da interação online. Foram excluídos os profissionais que não atuavam na área da oncologia.

Para a seleção dos participantes, utilizou-se a técnica snowball sampling (amostragem bola de neve) ${ }^{14}$. Para o desenvolvimento da estratégia bola de neve, foram contatados, por meio de correio eletrônico (e-mail), profissionais de saúde que prestam assistência a pessoas com câncer em contexto hospitalar. Esses profissionais ajudaram o pesquisador a iniciar seus contatos online e a buscar o grupo a ser pesquisado. Foi solicitado às pessoas indicadas que sugerissem novos contatos com as características desejadas por esta pesquisa, a partir de sua própria rede pessoal/profissional. Para a definição do número de participantes, utilizou-se a amostragem por saturação teórica ${ }^{15}$.

A coleta de dados ocorreu no período de março a agosto de 2017, utilizando um roteiro de entrevista semiestruturado constituído por questões sociodemográficas e por cinco questões norteadoras: 1. Quais motivos o (a) levaram a escolher trabalhar junto à pessoa com câncer? 2. Na sua perspectiva, qual o significado que você atribui ao câncer? 3. Quais sentimentos são despertados em você diante da morte de seu paciente com câncer? 4. Para você, quais dificuldades os profissionais de saúde enfrentam ao lidar com a morte de pacientes com câncer? 5 . Na sua perspectiva, qual o significado/sentido que você atribui à morte de uma pessoa com câncer que está sob seus cuidados? 
Cada entrevista teve duração aproximada de uma hora, em horários agendados pelo participante segundo a sua disponibilidade. Na entrevista, os participantes que desejaram ser entrevistados via chat, tiveram seus pedidos levados em consideração e todo conteúdo da entrevista ficou registrado por escrito. Para aqueles que optaram por videoconferência, foi utilizado um gravador digital portátil para registro com a ciência do participante. Suas falas foram transcritas na íntegra, além de serem verificadas duas vezes, por dois pesquisadores, para garantir sua fidedignidade.

Os dados foram transcritos e analisados por meio da técnica de análise de conteúdo temática, seguindo-se as fases de pré-análise, exploração do material, tratamento e interpretação dos resultados obtidos ${ }^{12}$. Na pré-análise foi realizada leitura flutuante do material proveniente das entrevistas com o objetivo de impregnação desse conteúdo pelo pesquisador. Na exploração do material, os temas foram processualmente agrupados conforme seus conteúdos, a partir das unidades de significado originadas do material, até se obter a formação de categorias. O tratamento e interpretação dos resultados obtidos foram articulados junto ao referencial teórico sobre a "Construção de Significados" do luto 8 .

A pesquisa foi aprovada pelo Comitê de Ética da instituição, sob parecer $n$ ㅇ 1.781.468. Para garantir o anonimato dos participantes, foram utilizadas letras para representar as seguintes categorias profissionais: E (enfermeiros), M (médicos), TO (terapeutas ocupacionais), P (psicólogos), F (fisioterapeutas), N (nutricionistas), seguidas do número sequencial das entrevistas.

\section{RESULTADOS E DISCUSSÃo}

Referente ao perfil sociodemográfico dos 34 participantes, têm-se as seguintes categorias profissionais: enfermeiros $(n=10)$, terapeutas ocupacionais $(n=8)$, médicos $(n=6)$, nutricionistas $(n=4)$, fisioterapeutas $(n=3)$ e psicólogos $(n=3)$. Suas idades variaram entre 24 e 49 anos, sendo a média de 30,32 anos. Dentre eles, 32 são do sexo feminino e dois do sexo masculino.

Em relação ao estado civil, 21 profissionais relataram serem solteiros, 11 casados, um divorciado e o restante em união estável. Quanto à crença religiosa, 17 declararam-se católicos, oito espíritas, três evangélicos, um budista e cinco referiram não pertencer a nenhuma religião.

Sobre o grau de escolaridade, todos os participantes possuíam nível superior e relataram que fizeram $(n=25)$ ou estão fazendo pós-graduação nas modalidades residência, especialização, mestrado ou doutorado ( $n=9$ ). Grande parte dos entrevistados $(n=19)$ possuía especialização na área da Oncologia. O tempo médio de experiência profissional nessa área foi de 4,01 anos. Um profissional da Medicina e outro da Terapia Ocupacional possuíam especialização na área de Cuidados Paliativos e um profissional da Nutrição treinamento nessa área.

A partir da análise dos dados, emergiram duas categorias: A morte como processo inerente à vida e término de sofrimento; Obstáculos a serem superados diante da morte do paciente na área da oncologia.

\section{A morte como processo inerente à vida e término do sofrimento}

A construção de significados diante do luto da vida humana é um processo que envolve encontrar um sentido/explicação para a perda, que está baseada em um modelo de crenças e visão de mundo ${ }^{8,16}$. Apesar da morte ser um fenômeno universal, cada pessoa pode atribuir-lhe um significado diferente, relacionado ao sentido encontrado/construído com base em suas experiências vividas, além de ser essencial para o ajustamento entre a pessoa enlutada e a perda vivida ${ }^{8}$.

Neste estudo, os profissionais de saúde que vivenciaram o processo de morte de seus pacientes com câncer atribuíram-lhe diferentes significados, sendo um deles como um processo inerente ao ciclo da vida humana:

Tento enxergar (a morte) como um processo natural [...] (N3).

Representa que o ciclo dele chegou ao fim e que todas as possibilidades terapêticas foram exauridas (E1).

Evidencia-se nesses relatos que à morte do paciente com câncer foi atribuído o sentido de um processo natural da vida. Alguns estudos apontam que muitos profissionais têm a tendência de naturalizar a noção da morte na tentativa de reduzir o desconforto em falar/ pensar a respeito dela ${ }^{17,18}$.

O sentido da morte do paciente com câncer mostrou-se também atrelado à vontade de um ser superior e de passagem para uma nova existência:

Quando são pacientes com diagnóstico que arrasta em cuidados paliativos, pedimos a Deus para que faça a sua vontade sempre [...] (E10).

[...] eu acredito em termos transcendentais que nossa vida atual é uma etapa (P1).

Posso pensar no sentido de passagem para outro local em que ele foi preparado para estar [...] (TO4).

As crenças e os valores religiosos desses participantes exerceram influência nos significados da morte e no processo de aceitação da perda de seus pacientes ${ }^{19}$. Destaca-se a importância de valorizar a espiritualidade dos 
profissionais de saúde para que possam considerá-la no cuidado aos pacientes em situação de terminalidade. Essa questão corrobora um estudo que constatou que a espiritualidade, para os profissionais da enfermagem, é uma dimensão importante da assistência paliativa, considerada uma fonte de força, conforto e fé, com impactos significativos na melhora do quadro clínico de pacientes, contribuindo para o enfrentamento desses indivíduos em seu processo de adoecimento e terminalidade ${ }^{20}$.

Quando o sofrimento do paciente com câncer fora das possibilidades terapêuticas de cura é intenso, a morte, comumente, passa ser significada como alívio da dor pela equipe de saúde. É uma forma que encontra para solucionar não só a dor vivenciada quando todas as possibilidades terapêuticas foram exauridas e, consequentemente, não se conseguiu salvar a vida desses indivíduos, mas também para protegê-los do sofrimento psíquico da perda do paciente ${ }^{21}$ :

Na finitude da vida, geralmente o paciente está sofrendo com dores, dispneia, e com a morte isso também tem um fim (E1).

Ao presenciarem a morte de seus pacientes com câncer são despertados nos profissionais de saúde diversos sentimentos:

Tristeza e impotência. Impotência por não ter conseguido o êxito de levar o paciente à cura (M2).

São diversos, tristeza, compaixão, impotência e frustração [...] (TO2).

Além desses sentimentos, na literatura evidencia incerteza, medo, desespero e ansiedade $2,3,18$

Em casos de maior vínculo entre profissional e paciente, a morte configurou-se como perda, intensificando o sentimento de tristeza e afetando esses profissionais, principalmente naqueles casos em que ocorrem a morte de pacientes jovens ou ainda crianças $^{22}$ :

Fico mais mobilizada com o paciente que construiu melhor vínculo comigo [...] (P1).

Alguns pacientes nos chocam mais, os mais jovens e as crianças [...] (E6).

Alguns profissionais, na tentativa de se protegerem e não vivenciarem sentimentos que podiam Ihes causar algum sofrimento, criaram mecanismos de enfrentamento durante o processo de morte de seus pacientes, procurando manter distância ou não se envolverem afetivamente com os mesmos ${ }^{4,5,23}$, como pode ser observado na seguinte fala:

Em outros casos de óbitos de pacientes com câncer, eu não fiquei chateada pelo distanciamento, com alguns pacientes eu me envolvi mais. Com outros a relação era muito profissional (N4).

Percebe-se que uma formação acadêmica voltada à reabilitação e restauração da saúde, com vistas ao prolongamento da vida e cura, gera nos profissionais uma percepção de insucesso/fracasso profissional diante da morte $^{3,23}$. É necessário oferecer formação profissional buscando prepará-los para lidarem com a morte nas suas histórias pessoais e em seu contexto de trabalho ${ }^{2,3,24}$.

Nota-se a importância da construção de espaços institucionais de compartilhamento de experiências, discussão e formação profissional relacionadas à finitude da vida, valorizando os significados que as equipes de saúde atribuem à morte de seus pacientes, que poderá ser uma forma de reduzir o estresse e as situações de sofrimento ${ }^{5,25}$.

Sobre a construção dos significados após a perda de uma pessoa querida, os enlutados têm que procurar não apenas um sentido para a morte ocorrida, mas também um novo sentido para suas próprias vidas, tornando a busca por um sentido e significado uma prioridade no processo de luto ${ }^{9}$. Esse movimento é importante, pois perdas que não fazem sentido e nas quais o indivíduo em luto não encontra significado acabam por desafiar o enlutado a reexaminar e reavaliar suas estruturas de significado prévias $^{11,26}$.

Entre sentidos/significados atribuídos em resposta à perda, destacam-se o encontro de benefícios e a transformação de identidade ${ }^{26}$. Observou-se que alguns participantes se mostraram impotentes e frustrados com a morte de seus pacientes. Entretanto, quando o enlutado pode apropriar-se de seu crescimento com essa experiência de perda, frequentemente se sente mais fortalecido, o que pode contribuir para a assimilação da mesma ${ }^{27}$. É possível notar um movimento de transformação da identidade, que implicará em um processo de reconstrução do próprio self; isto é, embora a dor e a angústia sejam esperadas, ela pode possibilitar uma identidade que busque maior empatia para com os pacientes, bem como crescimento pessoal e espiritual ${ }^{26}$. Assim, percebe-se que encontrar benefícios na experiência de perda de um ente querido e crescer com ela pode contribuir positivamente no processo de adaptação e elaboração dessa perda pelo enlutado ${ }^{9,26,27}$.

De acordo com estudos 9,11 , explorar os significados que as pessoas atribuem à morte de um ente querido e estimular a reflexão sobre os sentidos que constroem diante dessa perda, com base nas narrativas e experiências, são recursos terapêuticos que auxiliam tanto no processo de construção do luto quanto no alívio do sofrimento. Sendo de suma importância a compreensão dos significados que os profissionais atribuem à morte de seus pacientes, no intuito de auxiliá-los no processamento dessas perdas e fortalecimento emocional. 


\section{Obstáculos a serem superados diante da morte do paciente na área da oncologia}

Nessa categoria, observou-se que um dos sentidos atribuídos aos obstáculos diante da morte do paciente com câncer foi a carência de acompanhamento e suporte psicológico ao profissional de saúde para o enfrentamento do luto pessoal:

Não há suporte psicológico para os profissionais, a gente tem o suporte psicológico para os pacientes [...] (M3).

[...] falta de acolhimento a esse profissional no sentido de que pela rotina, principalmente hospitalar, serem raros os momentos de escuta a ele (TO4).

Dessa forma, é relevante a oferta do suporte psicológico para os profissionais da saúde, junto aos serviços, auxiliando-os a cuidarem de sua própria saúde mental quando lidam com a morte de seus pacientes ${ }^{28}$. Estudo destacou que o apoio de psicólogos junto a equipe de saúde foi considerado de extrema importância pelos profissionais, ajudando-os a enfrentarem situações que causam sofrimento no trabalho ${ }^{29}$. Outro estudo apontou que a oferta de práticas integrativas, tais como a Auriculoterapia e Reiki podem contribuir para a promoção da saúde mental desses profissionais ${ }^{30}$.

Percebe-se, a importância de uma cultura institucional que valorize a saúde física e mental desses profissionais, por meio da oferta de canais de discussão, rodas de conversa, educação permanente e serviços de psicologia, entre outras estratégias, que possam acolher e cuidar das equipes que lidam diariamente com o sofrimento do paciente com câncer e de seus familiares. Essas ações propiciarão não só benefícios aos profissionais da saúde, mas também uma melhor assistência ao paciente e à sua família ${ }^{5}$, fortalecendo a qualidade do cuidado.

Além do suporte psicológico, é crucial preparar esses profissionais para a comunicação de notícias difíceis e para a construção de ações voltadas à oferta de apoio e suporte aos pacientes e familiares. Os participantes reconhecem a importância de uma equipe multiprofissional para dar suporte à família enlutada:

Conversar com a família tanto antes do óbito quanto depois do óbito em dar a má notícia [...] (M2).

[...] falta de um acompanhamento multidisciplinar, eu não tenho preparo psicológico para acolher/orientar um familiar nesse momento (morte) [...] (M5).

Torna-se necessário que, durante a formação de profissionais da área da saúde, a comunicação de más notícias seja incluída como uma disciplina fundamental ${ }^{31,32}$. Um estudo de revisão sistemática constatou que as estratégias mistas envolvendo exercícios práticos e teóricos sobre a comunicação de más notícias, possibilitam melhora na capacidade comunicativa de estudantes/profissionais de saúde ${ }^{33}$.

Evidencia-se a importância de uma equipe interdisciplinar para favorecer a integralidade do cuidado, do paciente e de sua família. Esta articulação é imprescindível, visto que nenhuma ciência/especialidade, separadamente, consegue abranger a complexidade da existência humana, e, por isso, há a necessidade de equipes integradas para lidar com todas as dimensões e formas de cuidar, buscando a redução do sofrimento e da dor, e, consequentemente, a melhoria da qualidade de vida do paciente com câncer e de seus familiares ${ }^{34}$.

No cuidado da família que vivencia o processo de terminalidade de seu ente querido, a espiritualidade pode ser um recurso importante. Um estudo ${ }^{35}$ abordou sobre uma tecnologia leve de cuidado espiritual denominada "permissão de partida". Nela, é sugerido aos participantes que fiquem de mãos dadas junto ao paciente (consciente ou não), com o desenvolvimento de alguns passos: afirmação do paciente como ser humano digno de respeito; expressão de sentimentos; perdão das falhas cometidas; resgate de momentos felizes com o paciente que está partindo; cada familiar pode verbalizar de forma positiva algo que tenha marcado a existência junto à pessoa que está em processo de terminalidade, bem como dizer a ela que pode ir em paz e que os membros familiares irão cuidar uns dos outros; e leitura de uma mensagem ou de fazer uma oração junto à esta pessoa. Esse recurso tem se mostrado promissor, pois além de possibilitar uma relação terapêutica favorável e de confiança com o paciente e seus familiares, também pode diminuir o sofrimento espiritual desses indivíduos por facilitar a expressão livre de seus sentimentos, crenças e rituais religiosos ou espirituais frente à finitude.

Destaca-se que, apesar da maioria dos participantes terem especialização na área da oncologia, alguns deles atribuíram como obstáculo em lidar com a morte de seus pacientes a carência de capacitação a respeito de assuntos voltados à finitude da vida e cuidados paliativos:

Falta de capacitação, de aulas de como lidar com a morte (N2).

Se não estamos bem, fica bastante difícil lidar com a morte do paciente [...] principalmente pela falta de se discutir cuidados paliativos (TO4).

Tais fatos demonstram o quão importante é que as instituições ofereçam espaços de discussão e cursos, pois profissionais sensibilizados e formados para assistir pacientes em cuidados paliativos apresentam melhores resultados, 
como controle de sintomas físicos (dor), a atenção aos sofrimentos psicossociais, e o desenvolvimento de competências e habilidades específicas relacionadas ao cuidado no fim da vida ${ }^{36,37}$.

Observou-se que os participantes atribuíram diferentes significados à morte de seus pacientes com câncer, assim compreender esses significados é essencial para o desenvolvimento de ações e estratégias de enfrentamento. Além disso, uma reflexão sobre esses sentidos poderá refletir positivamente no cuidado em saúde, proporcionando às pessoas atendidas uma morte digna e humanizada, que leve em consideração seus anseios e desejos.

Assim, a criação de espaços de cuidado da saúde mental no trabalho por parte dos gestores é fundamental. Estratégias de suporte psicológico, inserção de práticas integrativas, oferta de cursos e/ou grupos de discussão sobre a temática da morte, cuidados paliativos e comunicação de más notícias, tanto de forma teórica quanto prática, podem ser efetivas na formação humana e profissional desses trabalhadores frente à terminalidade e à necessidade de apoio qualificado à família enlutada. Ademais, é de suma importância às instituições de ensino dar maior ênfase à temática da morte durante o processo de formação dos profissionais de saúde por meio de aulas expositivas, leituras reflexivas e situações práticas, com a finalidade de prepará-los a lidarem com o processo de finitude da vida humana.

Como limitação da pesquisa, sinaliza-se o envolvimento exclusivo de profissionais de saúde brasileiros que atuam na área da oncologia. Assim, o desenvolvimento de pesquisas com profissionais de outras nacionalidades é fundamental para ampliar o entendimento sobre a construção do significado da morte na diversidade cultural.

\section{CONCLUSÃO}

Constatou-se que os profissionais de saúde construíram diferentes significados da morte de seus pacientes com câncer, como um processo natural da vida humana, vontade de um ser superior, de passagem para uma nova existência, e fim do sofrimento, tanto do paciente quanto da equipe de saúde. Sugere-se, a criação de estratégias, como espaços de suporte psicológico e grupos de educação para morte, com a finalidade de cuidar da saúde desses trabalhadores.

\section{REFERÊNCIAS}

1. Torre LA, Siegel RL, Ward EM, Jemal A. Global cancer incidence and mortality rates and trends--an update. Cancer Epidemiol. Biomarkers Prev. [Internet]. 2016 [cited 2020 June 02]; 25(1):16-27. DOI: http://dx.doi.org/10.1158/1055-9965.EPI-15-0578.

2. Machado RS, Lima LAA, Silva GRF, Monteiro CFS, Rocha SS. Finitude and death in western society: a reflection focusing on health professionals. Cult. Cuid. [Internet]. 2016 [cited 2019 Dec 2]; 20(45):91-7. DOI: http://dx.doi.org/10.14198/cuid.2016.45.10.

3. Oliveira-Cardoso EA, Santos MA. Death education group: a strategy to complement undergraduate education of health professionals. Pscicol. Ciênc. Prof. [Internet]. 2017 [cited 2019 Dec 2]; 37(2):500-14. DOI: http://dx.doi.org/10.1590/19823703002792015.

4. Zheng R, Lee SF, Bloomer MJ. How nurses cope with patient death: A systematic review and qualitative meta-synthesis. J. Clin. Nurs. [Internet]. 2018 [cited 2020 Nov 20]; 27(1-2):e39-e49. DOI: https://doi.org/10.1111/jocn.13975.

5. Luz KR, Vargas OAM, Barlem ELD, Schmitt PH, Ramos FRS, Meirelles BHS. Coping strategies for oncology nurses in high complexity. Rev. Bras. Enferm. [Internet]. 2016 [cited 2019 Dec 2]; 69(1):59-63. DOI: http://dx.doi.org/10.1590/00347167.2016690109i.

6. Reis CGC, Farias CP, Quintana AM. Emptiness of sense: religiosity support for patients with advanced cancer. Pscicol. Ciênc. Prof. [Internet]. 2017 [cited 2019 Dec 2]; 37(1):106-18. DOI: http://dx.doi.org/10.1590/1982-3703000072015.

7. Luna IJ, Moré CO. Narratives and reconstruction of meaning in the bereavement process. Revista M [Internet]. 2017 [cited 2020 Nov 23]; 2(3):152-72. DOI: http://dx.doi.org/10.9789/2525-3050.2017.v2i3.152-172.

8. Neimeyer RA. Aprender de la pérdida: una guía para afrontar el duelo. Barcelona: Booket; 2019.

9. Neimeyer RA, Klass D, Dennis MR. A social constructionist account of grief: loss and the narration of meaning. Death Stud [Internet]. 2014 [cited 2019 Dec 2]; 38(6-10):485-98. DOI: http://dx.doi.org/10.1080/07481187.2014.913454.

10. Sánchez-Sánchez LM, López-Córdova ED, Siller-Gómez P. Attitude of the medical personnel of a pediatrics department towards patient death. Gac. Med. Mex. [Internet]. 2018 [cited 2020 June 2]; 154(2):152-160. DOI: http://dx.doi.org/10.24875/GMM.18002358.

11. Neimeyer RA. Meaning reconstruction in the wake of loss: evolution of a research program. Behaviour Change [Internet] 2016 Apr [cited 2019 Dec 2]; 33(2):65-79. DOI: https://doi.org/10.1017/bec.2016.4.

12. Minayo MCS. O desafio do conhecimento: pesquisa qualitativa em saúde. 14. ed. São Paulo (SP): Hucitec; 2014.

13. Flick U. Introdução à Pesquisa Qualitativa. 3. ed. Porto Alegre (RS): Artmed; 2009. p. 238-253.

14. Faugier J, Sargeant M. Sampling hard to reach populations. J. Adv. Nurs. [Internet]. 1997 Oct [cited 2019 Dec 2]; 26(4):790-7. DOI: https://doi.org/10.1046/j.1365-2648.1997.00371.x.

15. Fontanella BJB, Luchesi BM, Saidel MGB, Ricas J, Turato ER, Melo DG. Sampling in qualitative research: a proposal for procedures to detect theoretical saturation. Cad. Saúde Pública [Internet]. 2011 [cited 2019 Dec 2]; 27(2):388-94. DOI: http://dx.doi.org/10.1590/S0102-311X2011000200020. 
16. Santos MR, Wiegand DL, Sá NN, Misko MD, Szylit R. From hospitalization to grief: meanings parents assign to their relationships with pediatric oncology professional. Rev. Esc. Enferm. USP [Internet]. 2019 [cited 2020 Nov 20];53:e03521. DOI: http://dx.doi.org/10.1590/S1980-220X2018049603521.

17. Perboni JS, Zilli F, Oliveira SG. Health professionals and the patient death and die process: an integrating review. Pers. Bioét. [Internet]. 2018 Dec [cited 2020 Nov 22]; 22(2):288-302. DOI: http://dx.doi.org/10.5294/pebi.2018.22.2.7.

18. Guo $Q$, Zheng R. Assessing oncology nurses' attitudes towards death and the prevalence of burnout: a cross-sectional study. Eur. J. Oncol. Nurs. [Internet]. 2019 [cited 2020 June 2]; 42:69-75. DOI: http://dx.doi.org/10.1016/j.ejon.2019.08.002.

19. Barbosa AGC, Massaroni L, Lima, EFA. Meaning of the process of dying and death for multiprofessional staff. Rev. Pesqui. Cuid. Fundam. [Internet]. 2016 [cited 2019 Dec 2]; 8(2):4510-7. Available from: http://www.seer.unirio.br/index.php/cuidadofundamental/article/view/4849.

20. Evangelista CB, Lopes MEL, Costa SFG, Abrão FMS, Batista PSS, Oliveira RC. Spirituality in patient care under palliative care: a study with nurses. Esc. Anna Nery [Internet]. 2016 Mar [cited 2019 Dec 2]; 20(1):176-82. DOI: http://dx.doi.org/10.5935/14148145.20160023.

21. Zheng R, Lee SF, Bloomer MJ. How nurses cope with patient death: A systematic review and qualitative meta-synthesis. J. Clin. Nurs. [Internet]. 2018 [cited 2020 June 2]; 27(1-2):39-49. DOI: http://dx.doi.org/10.1111/jocn.13975.

22. Lima MJV, Andrade NM. The work of the resident health professional in contact with death and dying. Saúde Soc [Internet]. 2017 [cited 2019 Dec 2]; 26(4):958-72. DOI: http://dx.doi.org/10.1590/s0104-12902017163041.

23. Barbosa AMGC, Massaroni L. Living with death and dying. Rev. Enferm. UFPE On Line [Internet]. 2016 [cited 2019 Dec 2]; 10(2):457-63. DOI: https://periodicos.ufpe.br/revistas/revistaenfermagem/article/viewFile/10977/12314.

24. Braz MS, Franco MHP. Palliative care professionals and their contribution to the prevention of complicated grief. Pscicol. Ciênc. Prof. [Internet]. 2017 [cited 2019 Dec 2]; 37(1):90-105. DOI: http://dx.doi.org/10.1590/1982-3703001702016.

25. Cerit B. Influence of training on first-year nursing department students' attitudes on death and caring for dying patients: a single-group pretest-posttest experimental study. Omega (Westport) [Internet]. 2019 [cited 2020 June 2];78(4):335-47. DOI: http://dx.doi.org/10.1177/0030222817748838.

26. Gillies J, Neimeyer RA. A. J. Constr. Psychol. [Internet] 2006 Aug [cited 2019 May 16]; 19(1):31-65. DOI: https://doi.org/10.1080/10720530500311182.

27. Gillies JM, Neimeyer RA, Milman E. The grief and meaning reconstruction inventory (gmri): initial validation of a new measure. Death Stud [Internet]. 2015 [cited 2020 June 2];39(1-5):61-74. DOI: https://doi.org/10.1080/07481187.2014.907089.

28. Granek L, Ben-David M, Nakash O, Cohen M, Barbera L, Ariad S, et al. Oncologists' negative attitudes towards expressing emotion over patient death and burnout. Support Care Cancer [Internet]. 2017 [cited 2020 June 2]; 25(5):1607-14. DOI: http://dx.doi.org/10.1007/s00520-016-3562-y.

29. Bubolz BK, Barbosa MCN, Amaral DED, Viegas ACV, Bernandes LS, Muniz RM. Perceptions of nursing professionals with regards to the suffering and its coping strategies in oncology. Rev. Fund. Care Online [Internet]. 2019 Apr [cited 2020 Nov 22]; 11(3):599-606. DOI: http://www.seer.unirio.br/index.php/cuidadofundamental/article/view/6596/0.

30. Cunha JHS, Frizzo HCF, Souza LMP. Complementary therapies in the health care of professionals who care for people with cancer. Cad. Naturol. Terap. Complem. [Internet]. 2016 [cited 2020 Nov 22]; 5(9):69-74. DOI: http://dx.doi.org/10.19177/cntc.v5e9201669-74.

31. Bastos BR, Fonseca ACG, Pereira AKS, Silva LCS. Health care professionals' training in communicating bad news in oncologic palliative care. Rev. Bras. Cancerol. [Internet]. 2016 [cited 2019 Dec 2]; 62(3):263-6. DOI: https://doi.org/10.32635/21769745.RBC.2016v62n3.342.

32. Setubal MSV, Gonçalves AV, Rocha SR, Amaral EM. Breaking bad news training program based on video reviews and spikes strategy: what do perinatology residents think about it? Rev. Bras. Ginecol. Obstet. [Internet]. 2017 Oct [cited 2020 Nov 23]; 39(10):552-9. DOI: https://doi.org/10.1055/s-0037-1604490.

33. Camargo NC, Lima MG, Brietzke E, Mucci S, Góis AFT. Teaching how to deliver bad news: a systematic review. Rev. Bioét. [Internet]. 2019 June [cited 2020 Nov 22]; 27(2):326-40. DOI: https://doi.org/10.1590/1983-80422019272317.

34. Oliveira TCB, Maranhã TLG, Barroso ML. Multiprofessional pediatric oncology palliative care team: a systematic review. Id On Line Rev. Psic. [Internet]. 2017 [cited 2019 Dec 2]; 11(35):492-530. DOI: https://doi.org/10.14295/idonline.v11i35.754.

35. Zenevicz LT, Bitencourt JVOV, Léo MMF, Madureira VSF, Thofehrn MB. Permission for departing: spiritual nursing care in human finitude. Rev. Bras. Enferm. [Internet]. 2020 [cited 2020 Nov 21]; 73(3):e20180622. DOI: https://doi.org/10.1590/0034-71672018-0622.

36. Hui D, Hannon BL, Zimmermann C, Bruera E. Improving patient and caregiver outcomes in oncology: team-based, timely, and targeted palliative care. CA Cancer J. Clin [Internet]. 2018 [cited 2020 Nov 23]; 68(5):356-76. DOI: https://doi.org/10.3322/caac.21490.

37. Jiang $Q$, Lu Y, Ying Y, Zhao H. Attitudes and knowledge of undergraduate nursing students about palliative care: an analysis of influencing factors. Nurse Educ. Today [Internet]. 2019 [cited 2020 June 2]; 80:15-21. DOI: https://doi.org/10.1016/j.nedt.2019.05.040. 